$\mathrm{SPhT} / 00-009$

\title{
PRECISE DETERMINATION OF CRITICAL EXPONENTS AND EQUATION OF STATE BY FIELD THEORY METHODS
}

\author{
J. ZINN-JUSTIN \\ CEA-Saclay, Service de Physique Théorique*, F-91191 Gif-sur-Yvette, \\ Cedex, FRANCE \\ E-mail: zinn@spht.saclay.cea.fr
}

\begin{abstract}
Renormalization group, and in particular its Quantum Field Theory implementation has provided us with essential tools for the description of the phase transitions and critical phenomena beyond mean field theory. We therefore review the methods, based on renormalized $\phi_{3}^{4}$ quantum field theory and renormalization group, which have led to a precise determination of critical exponents of the $N$-vector model [1, 2] and of the equation of state of the 3D Ising model [3]. These results are among the most precise available probing field theory in a nonperturbative regime.

Precise calculations first require enough terms of the perturbative expansion. However perturbation series are known to be divergent. The divergence has been characterized by relating it to instanton contributions. The information about large order behaviour of perturbation series has then allowed to develop efficient "summation" techniques, based on Borel transformation and conformal mapping [4].

We first discuss exponents and describe our recent results [2]. Compared to exponents, the determination of the scaling equation of state of the 3D Ising model involves a few additional (non-trivial) technical steps, like the use of the parametric representation, and the order dependent mapping method. From the knowledge of the equation of state a number of ratio of critical amplitudes can also be derived.

Finally we emphasize that few physical quantities which are predicted by renormalization group to be universal have been determined precisely, and much work remains to be done. Considering the steady increase in the available computer
\end{abstract}

Talk given at the Conference "Renormalization Group 2000, Taxco (Mexico), 11-15 Jan. 1999

*Laboratoire de la Direction des Sciences de la Matière du Commissariat à l'Energie Atomique 
resources, many new calculations will become feasible. In addition to the infinite volume quantities, finite size universal quantities would also be of interest, to provide a more direct contact with numerical simulations. Let us also mention dynamical observables, a largely unexplored territory.

\section{Introduction: the effective $\phi^{4}$ field theory}

Second order phase transitions are continuous phase transitions where the correlation length diverges. Renormalization group (RG) arguments [5], as well as an analysis, near dimension four, of the most IR divergent terms appearing in the expansion around mean field theory [6], indicate that such transitions possess universal properties, i.e. properties independent to a large extent from the details of the microscopic dynamics. Moreover all universal quantities can be calculated from renormalizable quantum field theories which are local when the interactions are short range. For an important class of physical systems and models one is led to a $\phi^{4}$-like euclidean field theory with $O(N)$ symmetry. Among those let us mention statistical properties of polymers, liquid-vapour, binary mixtures, superfluid Helium, ferromagnetic transitions... We explain here how critical exponents and other universal quantities have been calculated with the help of renormalization group ideas and their implementation within the quantum field theory context [7].

The effective quantum field theory. Wilson's renormalization group shows that, at least in the neighbourhood of dimension four, the universal properties of statistical models which short range interactions and $O(N)$ symmetry can generically be described by an effective euclidean field theory, with an action $\mathcal{H}(\phi)$

$$
\left.\mathcal{H}(\phi)=\int \mathrm{d}^{d} x\left\{\frac{1}{2}[\nabla \phi(x)]^{2}+\frac{1}{2} r \phi^{2}(x)+\frac{1}{4 !} g_{0} \Lambda^{4-d}\left(\phi^{2}(x)\right)\right)^{2}\right\}
$$

where $r$ is a regular function of the temperature $T$. The parameter $\Lambda$ has the dimension of a mass and corresponds to the inverse microscopic scale. It also appears as a cut-off in the Feynman diagrams of the perturbative expansion.

For some value $r_{c}=r\left(T_{c}\right)$ the correlation length $\xi$ diverges (the physical mass $m=1 / \xi$ vanishes). Near the critical temperature $T_{c}$, in the critical domain $\left|T-T_{c}\right| \propto\left|r-r_{c}\right| \ll \Lambda^{2},|\langle\phi\rangle| \ll \Lambda^{(d-2) / 2}$, the mass remains small, $m \ll \Lambda$. In this limit a universal behaviour of thermodynamic quantities is expected. The study of the critical domain thus reduces to the study of the large cut-off behaviour, i.e. to renormalization theory and the corresponding renormalization group. However, in the traditional presentation of quantum field theory in the context of particle physics, the dependence of the parameters of the action as a function of the cut-off $\Lambda$ is determined by the condition that renormalized correlation functions should have a finite cut-off limit. Here instead the coefficient 
$g_{0} \Lambda^{4-d} / 4$ ! of the $\phi^{4}$ interaction has a dependence on $\Lambda$ given a priori. In particular in the dimensions of interest, $d<4$, the "bare" coupling constant diverges for $\Lambda \rightarrow \infty$, a reflection of the IR instability of the gaussian fixed point, though the field theory, being super-renormalizable, requires only a mass renormalization.

The divergence of the bare coupling constant. One solution to the problem of the large coupling constant is provided by Wilson-Fisher's famous $\varepsilon$-expansion [8]. One defines, at least in perturbation theory, the field theory in arbitrary complex space dimension $d$. Setting $d=4-\varepsilon$ one then expands both in $g_{0}$ and $\varepsilon$. The large cut-off divergences become logarithmic as in four dimensions, and can be removed by standard renormalizations. Renormalization group (RG) equations for correlation functions follow, from which scaling laws can be derived, and which lead to calculations of physical quantities as power series in $\varepsilon$.

The most convenient RG equations are homogeneous differential equations, first derived for the critical (massless) theory [9], and then generalized to the whole critical domain $r \neq r_{c}$ [10]. They correspond to a scheme in which the massless (or critical) theory has a perturbative expansion in $g_{0}$ and therefore rely on the double series expansion in $g_{0}$ and $\varepsilon$. An alternative formalism is based on the Callan-Symanzik (CS) (inhomogeneous) equations for the massive theory [11]. One introduces correlation functions for a renormalized field $\phi_{\mathrm{R}}=Z^{-1 / 2} \phi$, expressed in terms of the physical mass $m$ or inverse correlation length $\xi^{-1}=m$ and a renormalized coupling constant $g$. They are implicitly defined by the conditions (after factorization of the obvious group indices):

$$
\begin{aligned}
\tilde{\Gamma}_{\mathrm{R}}^{(2)}(p ; m, g) & =m^{2}+p^{2}+O\left(p^{4}\right), \\
\tilde{\Gamma}_{\mathrm{R}}^{(4)}\left(p_{i}=0 ; m, g\right) & =m^{4-d} g,
\end{aligned}
$$

where the functions $\tilde{\Gamma}_{\mathrm{R}}^{(n)}$ are the Fourier transforms of so-called (1PI) correlation functions $\Gamma_{\mathrm{R}}^{(n)}$, the coefficients of the expansion of the thermodynamic potential in terms of the local field expectation value $\left\langle\phi_{\mathrm{R}}(x)\right\rangle$.

To prove scaling laws from the CS equations it remains necessary to expand in $g$ and $\varepsilon$. The form of the CS $\beta$-function in dimension $d$ is

$$
\beta(g)=-(4-d) g+a_{2}(d) g^{2}+\cdots, \quad a_{2}(d)>0 .
$$

For $\varepsilon=4-d$ small it has a zero $g^{*}$ of order $\varepsilon$ and one recovers the principle of the $\varepsilon$-expansion.

However in this framework, because the theory now is massive, the perturbative expansion is IR finite in any dimension and the CS equations are always satisfied. Moreover one generally assumes that the results established for the $\varepsilon$-expansion remain valid at finite $\varepsilon$. Therefore Parisi [12] has suggested working at fixed dimension $d<4$, in the massive theory (the massless theory is IR 
divergent). In contrast with the $\varepsilon$-expansion however, at fixed dimensions three or two no small parameter is available. Therefore a precise determination of $g^{*}$ and all other physical quantities depends on the analytic properties of the series, in addition to the number of terms available. A semi-classical analysis, based on instanton calculus, unfortunately indicates that perturbation in quantum field theory is always divergent. Therefore to extract any information from perturbation theory a summation method is required. Note finally that at fixed dimension, at any finite order, universal quantities are given by expressions which depend on the renormalization scheme, in contrast with the results of the $\varepsilon$-expansion.

This approach, which is more cumbersome, has eventually been tried for practical reasons: it is easier to calculate Feynman diagrams in dimension three than in generic dimensions, and thus more perturbative orders could be obtained.

While this approach is a natural extension of the $\varepsilon$-expansion, its interpretation directly at fixed dimension is worth discussing. At fixed dimension $d<4$, in the critical domain (condition which implies shifting $r$ by $r_{c}$ and thus performing a mass renormalization), all terms in the perturbative expansion have a finite large cut-off limit at $u_{0}=g_{0} \Lambda^{4-d}$ fixed because the theory is super-renormalizable. This means physically that the initial parameters of the field theory are first tuned to remain artificially close to the unstable $u_{0}=0$ gaussian fixed point. Indeed the true expansion parameter is a dimensionless quantity and therefore is proportional to $g_{0} \Lambda^{4-d} / m^{4-d}$. To keep this parameter constant when $m$ the physical mass goes to zero for $T \rightarrow T_{c}\left(r \rightarrow r_{c}\right)$ in the high temperature phase, one must vary the dimensionless parameter $g_{0}$ as

$$
g_{0}^{1 /(4-d)} \propto m / \Lambda \propto 1 / \xi \Lambda .
$$

The relevant theory, however, corresponds to the infinite $u_{0}$ limit. One is then confronted with a serious technical problem: perturbation theory is finite in the critical domain but one is interested in the infinite coupling limit, where obviously the perturbative expansion is no longer useful.

One thus introduces the renormalized coupling constant $g$ and the field renormalization defined by the conditions (1.2). Then

$$
u_{0}=g_{0} \Lambda^{(4-d)}=m^{(4-d)} G(g), \quad \beta(g)=(d-4) G(g) / G^{\prime}(g) .
$$

When the initial coupling constant $u_{0}$ becomes large the new dimensionless coupling $g$ has a finite limit provided the $\beta$-function has an IR stable zero $g^{*}$ :

$$
u_{0} \rightarrow \infty \Rightarrow \beta\left(g^{*}\right)=0 \quad \text { and } \omega \equiv \beta^{\prime}\left(g^{*}\right)>0 .
$$

In such a situation the renormalized coupling $g$ is a more suitable expansion parameter then $u_{0}$. 
The relations (1.3) then imply that at $g_{0}$ fixed, $\Lambda / m \rightarrow \infty$,

$$
g-g^{*} \propto(m / \Lambda)^{\omega} .
$$

To the field renormalization $Z(g)$ is associated the RG function $\eta(g)$

$$
\eta(g)=\beta(g) Z^{\prime}(g) / Z(g) .
$$

Integration of the equation (1.5) yields the behaviour of $Z(g)$ near $g^{*}$

$$
Z(g) \propto\left(g^{*}-g\right)^{\eta / \omega} \propto(m / \Lambda)^{\eta},
$$

where the exponent $\eta=\eta\left(g^{*}\right)$ characterizes the field anomalous dimension $d_{\phi}$ : $2 d_{\phi}=d-2+\eta$. This singular behaviour, consistent with the scaling properties derived from the $\varepsilon$-expansion, explains why even in dimension $d<4$ the introduction of a field renormalization is necessary.

The approach has sometimes be questioned, because it involves this double limit, but the final results and their comparison with other data have shown the consistency of the method.

A last remark: in this framework the mass parameter $m$ has still to be related to the temperature. RG equations show that it is singular at $T_{c}$ and behaves for $t=r-r_{c} \rightarrow 0_{+}$as

$$
m \propto t^{\nu} \propto\left(T-T_{c}\right)^{\nu},
$$

where $\nu$ is the correlation length exponent.

\section{Critical exponents and series summation}

Critical exponents are the most studied quantities in the theory of phase transitions, because they characterize universality classes and are easier to calculate. They have been extensively used to test RG predictions by comparing them with other results (experiments, high or low temperature series expansion, Monte-Carlo simulations) [13]. The first precise determination of the exponents of the $O(N)$ symmetric $N$-vector model has been reported by Le Guillou-ZinnJustin [1], using the six-loop series for RG functions calculated by Nickel et al [14]. The summation methods used in [1] were based on a Borel transformation

and conformal map. The same ideas have later been applied to the $\varepsilon$-expansion [15] when five loop series became available [16], and more recently to the equation of state by Guida-Zinn-Justin [3]. With time the method has been refined and the efficiency improved by various tricks but the basic principles have not changed. 


\subsection{Borel transformation and conformal mapping}

Let $F(g)$ be any quantity given by a perturbative series

$$
F(g)=\sum_{k=0} F_{k} g^{k} .
$$

A semi-classical analysis of barrier penetration effects for negative coupling (instantons) teaches us that in the $\phi_{3}^{4}$ field theory for large order $k$ the coefficients $F_{k}$ behave like

$$
F_{k} \underset{k \rightarrow \infty}{\propto} k^{s}(-a)^{k} k ! .
$$

The value of $a>0$ has been determined numerically (while $s$ is known analytically). One then introduces $B(b, g)$, the Borel (rather Borel-Leroy) transform of $F(g)$, which is defined by

$$
B(b, g)=\sum_{k=0} \frac{F_{k}}{\Gamma(b+k+1)} g^{k},
$$

where $b$ is a free real parameter $(b>-1)$. Formally, i.e. in the sense of series expansion, $F(g)$ can be recovered from

$$
F(g)=\int_{0}^{\infty} t^{b} \mathrm{e}^{-t} B(b, g t) \mathrm{d} t .
$$

Using the large order estimate in (2.2) one verifies that $B(b, z)$ is analytic at least in a circle of radius $1 / a$ and its singularity closest to the origin is located at $z=-1 / a$. Therefore $B(b, z)$, in contrast with $F(z)$, is determined by its series expansion. However, the relation (2.3) can be extended from a relation between formal series to a relation between functions only if $B(b, z)$ is analytic in a neighbourhood of the real positive axis. In the case of the $\phi_{3}^{4}$ field theory such a property has been proven in constructive field theory (as well as the property that the function $F(g)$ is indeed by given by (2.3)) [17. Moreover it is necessary to construct an analytic continuation of the function $B(b, z)$ from the circle to the real positive axis. Considerations about more general instanton contributions strongly suggest that the Borel transform actually is analytic in a cut-plane, the cut being on the real negative axis, at the left of $-1 / a$. Therefore an analytic continuation is provided by a conformal map of the cut-plane onto a circle:

$$
z \mapsto u(z)=\frac{\sqrt{1+a z}-1}{\sqrt{1+z a}+1} .
$$

The function $B[b, z(u)]$ is then given by a series in powers of $u$ convergent in the cut-plane. The corresponding (hopefully convergent) series expansion for function $F(g)$ takes the form

$$
F(g)=\sum_{k=0}^{\infty} U_{k}(b) \int_{0}^{\infty} t^{b} \mathrm{e}^{-t}[u(g t)]^{k} \mathrm{~d} t .
$$


The parameter $b$, as well as a few other parameters introduced in variants, are used to improve the apparent convergence and test the sensitivity of results to their variations. Moreover the value of $b$ has to stay within a reasonable range around the value $s$ predicted by the large order behaviour. Finally the summation method is expected to be efficient mainly when the available coefficients $F_{k}$ behave already as predicted by the asymptotic large order estimate.

\subsection{Exponents}

The values of critical exponents obtained from field theory have remained after about twenty years among the most precise determinations. Only recently have consistent, but significantly more precise, experimental results been reported in low gravity superfluid experiments [41]. Also the precision of results coming from high temperature expansions [18 29] and various numerical simulations [30 40] on the lattice has kept steadily improving.

Recently seven-loop terms have been obtained for $0 \leq N \leq 3$ for two of the three RG functions, related to the $\phi$ and $\phi^{2}$ dimensions, by Murray and Nickel 42]. These terms, together with some improvement in the summation methods, have led to the new slightly more precise values of $g^{*}$ and critical exponents displayed in table $1\left(\tilde{g}_{N i}^{*}=(N+8) g^{*} / 48 \pi\right)$ (Guida-Zinn-Justin [2]). Among the exponents $\gamma, \nu, \eta, \beta, \alpha$, only two are independent, for example

$$
\gamma=\nu(2-\eta), \quad \beta=\frac{1}{2} \nu(1+\eta), \quad \alpha=2-3 \nu,
$$

but they are calculated independently to test the precision of the summation method.

The main improvements concern the exponent $\eta$ which was poorly determined, and the lower value of $\gamma$ for $N=0$ (polymers).

In the framework of the $\varepsilon$-expansion, since the series used earlier were affected

by a small error at order $\varepsilon^{5}$, the values have also been updated (table 2). Two kinds of results are reported, free means simple summation as above, bc means that the known values in two dimensions have been incorporated in the summation procedure. It is gratifying that the overall consistency between the two set of values coming from $3 \mathrm{D}$ series and $\varepsilon$-expansion has improved.

Finally $O(4)$ results of interest for simulations of the Higgs phase transition at finite temperature, obtained from six loop series, have been added (table 3).

\section{The scaling equation of state}

Let us first recall a few properties of the equation of the state in the critical domain, in the specific case $N=1$ (Ising-like systems), at $d<4$.

The equation of state is the relation between magnetic field $H$, magnetization $M=\langle\phi\rangle$ (the "bare" field expectation value) and the temperature which is 
Table 1

Critical exponents of the $O(N)$ models from $d=3$ expansion [2].

\begin{tabular}{|c|c|c|c|c|}
\hline$N$ & 0 & 1 & 2 & 3 \\
\hline$\tilde{g}_{\mathrm{Ni}}^{*}$ & $1.413 \pm 0.006$ & $1.411 \pm 0.004$ & $1.403 \pm 0.003$ & $1.390 \pm 0.004$ \\
$g^{*}$ & $26.63 \pm 0.11$ & $23.64 \pm 0.07$ & $21.16 \pm 0.05$ & $19.06 \pm 0.05$ \\
$\gamma$ & $1.1596 \pm 0.0020$ & $1.2396 \pm 0.0013$ & $1.3169 \pm 0.0020$ & $1.3895 \pm 0.0050$ \\
$\nu$ & $0.5882 \pm 0.0011$ & $0.6304 \pm 0.0013$ & $0.6703 \pm 0.0015$ & $0.7073 \pm 0.0035$ \\
$\eta$ & $0.0284 \pm 0.0025$ & $0.0335 \pm 0.0025$ & $0.0354 \pm 0.0025$ & $0.0355 \pm 0.0025$ \\
$\beta$ & $0.3024 \pm 0.0008$ & $0.3258 \pm 0.0014$ & $0.3470 \pm 0.0016$ & $0.3662 \pm 0.0025$ \\
$\alpha$ & $0.235 \pm 0.003$ & $0.109 \pm 0.004$ & $-0.011 \pm 0.004$ & $-0.122 \pm 0.010$ \\
$\omega$ & $0.812 \pm 0.016$ & $0.799 \pm 0.011$ & $0.789 \pm 0.011$ & $0.782 \pm 0.0013$ \\
$\theta=\omega \nu$ & $0.478 \pm 0.010$ & $0.504 \pm 0.008$ & $0.529 \pm 0.009$ & $0.553 \pm 0.012$ \\
\hline
\end{tabular}

Table 2

Critical exponents of the $O(N)$ models from $\varepsilon$-expansion [2].

\begin{tabular}{|l|l|l|l|l|}
\hline \multicolumn{1}{|c|}{$N$} & \multicolumn{1}{|c|}{0} & \multicolumn{1}{c|}{1} & \multicolumn{1}{c|}{2} & \multicolumn{1}{c|}{3} \\
\hline$\gamma$ (free) & $1.1575 \pm 0.0060$ & $1.2355 \pm 0.0050$ & $1.3110 \pm 0.0070$ & $1.3820 \pm 0.0090$ \\
$\gamma$ (bc) & $1.1571 \pm 0.0030$ & $1.2380 \pm 0.0050$ & 1.317 & 1.392 \\
$\nu$ (free) & $0.5875 \pm 0.0025$ & $0.6290 \pm 0.0025$ & $0.6680 \pm 0.0035$ & $0.7045 \pm 0.0055$ \\
$\nu$ (bc) & $0.5878 \pm 0.0011$ & $0.6305 \pm 0.0025$ & 0.671 & 0.708 \\
$\eta$ (free) & $0.0300 \pm 0.0050$ & $0.0360 \pm 0.0050$ & $0.0380 \pm 0.0050$ & $0.0375 \pm 0.0045$ \\
$\eta$ (bc) & $0.0315 \pm 0.0035$ & $0.0365 \pm 0.0050$ & 0.0370 & 0.0355 \\
$\beta$ (free) & $0.3025 \pm 0.0025$ & $0.3257 \pm 0.0025$ & $0.3465 \pm 0.0035$ & $0.3655 \pm 0.0035$ \\
$\beta$ (bc) & $0.3032 \pm 0.0014$ & $0.3265 \pm 0.0015$ & & \\
$\omega$ & $0.828 \pm 0.023$ & $0.814 \pm 0.018$ & $0.802 \pm 0.018$ & $0.794 \pm 0.018$ \\
$\theta$ & $0.486 \pm 0.016$ & $0.512 \pm 0.013$ & $0.536 \pm 0.015$ & $0.559 \pm 0.017$ \\
\hline
\end{tabular}

represented by the parameter $t=r-r_{c} \propto T-T_{c}$. It is related to the free energy per unit volume, in field theory language the generating functional $\Gamma(\phi)$ of $1 \mathrm{PI}$ correlation functions restricted to constant fields, i.e the effective potential $\mathcal{V}$, $\mathcal{V}(M)=\Gamma(M) /$ vol., by $H=\partial \mathcal{V} / \partial M$. In the critical domain the equation of state has Widom's scaling form $(\delta=(d+2-\eta) /(d-2+\eta))$

$$
H(M, t)=M^{\delta} f\left(t / M^{1 / \beta}\right)
$$

a form initially conjectured and which renormalization group has justified.

One property of the function $H(M, t)$ which plays an essential role in the 
Table 3

Critical exponents in the $O(4)$ models from $d=3$ and $\varepsilon$-expansion [9].

\begin{tabular}{|c|c|c|}
\hline & $d=3$ & $\varepsilon:$ free, bc \\
\hline$\tilde{g}_{\mathrm{Ni}}^{*}$ & $1.377 \pm 0.005$ & \\
$g^{*}$ & $17.30 \pm 0.06$ & \\
$\gamma$ & $1.456 \pm 0.010$ & $1.448 \pm 0.015,1.460$ \\
$\nu$ & $0.741 \pm 0.006$ & $0.737 \pm 0.008,0.742$ \\
$\eta$ & $0.0350 \pm 0.0045$ & $0.036 \pm 0.004,0.033$ \\
$\beta$ & $0.3830 \pm 0.0045$ & $0.3820 \pm 0.0025$ \\
$\alpha$ & $-0.223 \pm 0.018$ & $-0.211 \pm 0.024$ \\
$\omega$ & $0.774 \pm 0.020$ & $0.795 \pm 0.030$ \\
$\theta$ & $0.574 \pm 0.020$ & $0.586 \pm 0.028$ \\
\hline
\end{tabular}

analysis is Griffith's analyticity: it is regular at $t=0$ for $M>0$ fixed, and simultaneously it is regular at $M=0$ for $t>0$ fixed.

Amplitude ratios. Universal amplitude ratios are numbers characterizing the behaviour of thermodynamical quantities near $T_{c}$. Several of them commonly considered in the literature can be directly derived from the scaling equation of state. Let us just give two examples.

The singular part of the specific heat, i.e. the $\phi^{2}$ two-point correlation function at zero momentum, behaves like

$$
C_{H}=A^{ \pm}|t|^{-\alpha}, \quad t \propto T-T_{c} \rightarrow \pm 0 .
$$

The ratio $A^{+} / A^{-}$then is universal.

The magnetic susceptibility $\chi$ in zero field, i.e. the $\phi$ two-point function at zero momentum, diverges like

$$
\chi=C^{ \pm}|t|^{-\gamma}, \quad t \rightarrow \pm 0
$$

The ratio $C^{+} / C^{-}$is also universal.

The $\varepsilon$-expansion. The first results concerning the scaling equation of state have been obtained within the framework of the $\varepsilon=4-d$ expansion.

The $\varepsilon$-expansion of the scaling equation of state has been determined up to order $\varepsilon^{2}$ for the general $O(N)$ model 43], and order $\varepsilon^{3}$ for $N=1$ [44]. We give here the function $f\left(x=t / M^{1 / \beta}\right)$ of eq. (3.1) for $N=1$ up to order $\varepsilon^{2}$ to display its structure,

$$
f(x)=1+x+\frac{1}{6} \varepsilon(x+3) L+\varepsilon^{2}\left[\frac{1}{72}(x+9) L^{2}+\frac{25}{324}(x+3) L\right]+O\left(\varepsilon^{3}\right),
$$


with $L=\log (x+3)$.

The expression (3.4) is not valid for $x$ large, i.e. for small magnetization $M$. In this regime the magnetic field $H$ has a regular expansion in odd powers of $M$. It is thus convenient to express the equation of state in terms of another scaling variable $z \propto x^{-\beta}$ because

$$
z \propto M t^{-\beta} .
$$

The equation of state then takes the form

$$
H \propto t^{\beta \delta} F(z),
$$

where the relation between exponents $\gamma=\beta(\delta-1)$ has been used. Substituting into eq. (3.4) $x=x_{0} z^{-1 / \beta}$ (the constant $x_{0}$ takes care of the normalization of $z$ ) and expanding in $\varepsilon$ one finds at order $\varepsilon^{2}$ for the function (3.6)

$$
F(z)=\tilde{F}_{0}(z)+\varepsilon \tilde{F}_{1}(z)+\varepsilon^{2} \tilde{F}_{2}(z)+O\left(\varepsilon^{3}\right),
$$

with

$$
\begin{aligned}
& \tilde{F}_{0}=z+\frac{1}{6} z^{3} \\
& \tilde{F}_{1}=\frac{1}{12}\left(-z^{3}+\tilde{L}\left(2 z+z^{3}\right)\right) \\
& \tilde{F}_{2}=\frac{1}{1296}\left(-50 z^{3}+\tilde{L}\left(100 z-4 z^{3}\right)+\tilde{L}^{2}\left(18 z+27 z^{3}\right)\right)
\end{aligned}
$$

and $\tilde{L}=\log \left(1+z^{2} / 2\right)$.

Within the framework of the formal $\varepsilon$-expansion one can easily pass from one expansion to the other. Still a matching problem arises if one wants to use the $\varepsilon$-expansion to determine the equation of state for $d=3$, i.e. $\varepsilon=1$. One is thus naturally led to look for a uniform representation valid in both limits. Josephson-Schofield parametric representation [45] has this property.

\section{Parametric representation of the equation of state}

In order to implement both Griffith's analyticity and the scaling relation, one parametrizes the equation of state in terms of two new variables $R$ and $\theta$, setting:

$$
\left\{\begin{aligned}
M & =m_{0} R^{\beta} \theta, \\
t & =R\left(1-\theta^{2}\right), \\
H & =h_{0} R^{\beta \delta} h(\theta),
\end{aligned}\right.
$$

where $h_{0}, m_{0}$ are two normalization constants. We choose $h_{0}$ such that

$$
h(\theta)=\theta+O\left(\theta^{3}\right) .
$$


In terms of the scaling variables $x$ of eq. (3.4) or $z$ from eq. (3.5) this parametrization corresponds to set

$$
\begin{aligned}
& z=\rho \theta /\left(1-\theta^{2}\right)^{\beta}, \quad \theta>0, \\
& x=x_{0} \rho^{-1 / \beta}\left(1-\theta^{2}\right) \theta^{-1 / \beta},
\end{aligned}
$$

where $\rho$ is some other positive constant.

Then the function $h(\theta)$ is an odd function of $\theta$ which from Griffith's analyticity is regular near $\theta=1$, which is $x$ small, and near $\theta=0$ which is $x$ large. It vanishes for $\theta=\theta_{0}$ which corresponds to the coexistence curve $H=0, T<T_{c}$.

Note that the mapping (4.2) is not invertible for values of $\theta$ such that $z^{\prime}(\theta)=0$. The derivative vanishes for $\theta=1 / \sqrt{(1-2 \beta)} \approx 1.69$. One has to verify that this value is reasonably larger than $\theta_{0}$, the largest possible value of $\theta$.

Finally it is useful for later purpose to write more explicitly the relation between the function $F(z)$ of eq. (3.6) and the function $h(\theta)$ :

$$
h(\theta)=\rho^{-1}\left(1-\theta^{2}\right)^{\beta \delta} F(z(\theta)) .
$$

Expanding both functions

$$
\begin{aligned}
F(z) & =z+\frac{1}{6} z^{3}+\sum_{l=2} F_{2 l+1} z^{2 l+1}, \\
h(\theta) / \theta & =1+\sum_{l=1} h_{2 l+1} \theta^{2 l}
\end{aligned}
$$

one finds the relations

$$
\begin{aligned}
h_{3}= & \frac{1}{6} \rho^{2}-\gamma \\
h_{5}= & \frac{1}{2} \gamma(\gamma-1)+\frac{1}{6}(2 \beta-\gamma) \rho^{2}+F_{5} \rho^{4} \\
h_{7}= & \frac{1}{6} \gamma(\gamma-1)(\gamma-2)+\frac{1}{12}(2 \beta-\gamma)(2 \beta-\gamma+1) \rho^{2} \\
& +(4 \beta-\gamma) F_{5} \rho^{4}+F_{7} \rho^{6}
\end{aligned}
$$

From the parametric representation of the equation of state it is then possible to derive a representation for the singular part of the free energy per unit volume as well as various universal ratios of amplitudes.

Parametric representation and $\varepsilon$-expansion. Up to order $\varepsilon^{2}$ the constant $m_{0}$ (or $\rho$ ) can be chosen in such a way that the function $h(\theta)$ reduces to:

$$
h(\theta)=\theta\left(1-\frac{2}{3} \theta^{2}\right)+O\left(\varepsilon^{2}\right) .
$$

The minimal model in which $h(\theta)$ is approximated by a cubic odd function of $\theta$ is called the linear parametric model. At order $\varepsilon^{2}$ the linear parametric model 
is exact, but at order $\varepsilon^{3}$ the introduction of a term proportional to $\theta^{5}$ becomes necessary [44,46]. One finds:

$$
h(\theta)=\theta\left(1+h_{3} \theta^{2}+h_{5} \theta^{4}\right)+O\left(\varepsilon^{4}\right),
$$

with

$$
h_{3}=-\frac{2}{3}\left(1+\frac{\varepsilon^{2}}{12}\right), \quad h_{5}=\frac{\varepsilon^{3}}{27}\left(\zeta(3)-\frac{1}{2} \lambda-\frac{1}{4}\right),
$$

where $\lambda$ is the constant

$$
\lambda=\frac{1}{3} \psi^{\prime}(1 / 3)-\frac{2}{9} \pi^{2}=1.17195361934 \ldots .
$$

The function $h(\theta)$ vanishes on the coexistence curve for $\theta=\theta_{0}$ :

$$
\theta_{0}^{2}=\frac{3}{2}\left(1-\frac{\varepsilon^{2}}{12}\right)+O\left(\varepsilon^{3}\right)
$$

Note that $h_{3}$ and thus $\theta_{0}$ are determined only up to order $\varepsilon^{2}$. It follows

$$
\rho^{2}=6\left(\gamma+h_{3}\right)=2\left(1+\frac{1}{2} \varepsilon+\frac{7}{108} \varepsilon^{2}\right)=3.13 \pm 0.13
$$

because $h_{3}$ is determined only up to order $\varepsilon^{2}$,

Remark. In the more general $O(N)$ case, the parametric representation also automatically generates equations of state with satisfy the required regularity properties, and thus leads to uniform approximations. However for $N>1$ the function $h(\theta)$ still has a singularity on the coexistence curve, due to the presence of Goldstone modes in the ordered phase and has therefore a more complicated form. The nature of this singularity can be obtained from the study of the non-linear $\sigma$-model. It is not clear whether a simple polynomial approximation would be useful. For $N=1$ instead, one expects at most an essential singularity on the coexistence curve, due to barrier penetration, which is much weaker and non-perturbative in the small $\varepsilon$ - or small $g$-expansion.

\section{Perturbative expansion at fixed dimension three}

We now discuss the calculations based on a perturbative expansion at fixed dimension $d=3$. Five loop series for the renormalized effective potential of the $\phi_{3}^{4}$ theory have been first reported in Bagnuls et al. [47], but the printed tables contain some serious misprints. These have been noticed by Halfkann and Dohm who have published corrected values [48]. These five-loop calculations have only been performed for $N=1$, because they are much more difficult for $N \neq 1$ due to the presence of two lengths, the correlation lengths along the applied field and transverse to it. 


\subsection{General remarks}

The general framework again is the massive theory renormalized at zero momentum. The correlation functions $\Gamma_{\mathrm{R}}^{(n)}$ of the renormalized field $\phi_{\mathrm{R}}=\phi / \sqrt{Z}$ are fixed by the normalization conditions (1.2). Eventually the renormalized coupling constant $g$ has to be set to its IR fixed point value $g^{*}$.

The conditions (1.2) imply that the free energy $\mathcal{F}$ expressed in terms of the "renormalized" magnetization $\varphi$, i.e. the expectation value of the renormalized field $\varphi=\left\langle\phi_{\mathrm{R}}\right\rangle$, has a small $\varphi$ expansion of the form (in $d$ dimensions)

$$
\mathcal{F}(\varphi)=\mathcal{F}(0)+\frac{1}{2} m^{2} \varphi^{2}+\frac{1}{4 !} m^{4-d} g \varphi^{4}+O\left(\varphi^{6}\right) .
$$

It is important to remember that the finite field renormalization $Z(g)$ is singular at $g^{*}$ (equation (1.6)). It follows that $\varphi \propto M / m^{\eta / 2}$.

It is convenient to introduce the rescaled variable $z$

$$
z=\varphi m^{(2-d) / 2} \sqrt{g} \propto M / m^{(d-2+\eta) / 2} \propto M / t^{\beta},
$$

(equation (1.7)) and set

$$
\mathcal{F}(\varphi)-\mathcal{F}(0)=\frac{m^{d}}{g} \mathcal{V}(z, g) .
$$

Taking into account the definitions of section 3, we conclude that the equation of state is related to the derivative $F$ of the reduced effective potential $\mathcal{V}$ with respect to $z$

$$
F(z, g)=\frac{\partial \mathcal{V}(z, g)}{\partial z}
$$

by

$$
H \propto t^{\beta \delta} F(z) .
$$

Ising symmetry implies that $F$ is an odd function of $z$

$$
F(z, g)=z+\frac{1}{6} z^{3}+\sum_{l=2} F_{2 l+1}(g) z^{2 l+1} .
$$

\subsection{The problem of the low temperature phase}

To determine the equation of state in the whole physical range, or universal ratios of amplitudes, a new problem arises. In this framework it is more difficult to calculate physical quantities in the ordered phase because the theory is parametrized in terms of the disordered phase correlation length $\xi=m^{-1} \propto$ $\left(T-T_{c}\right)^{-\nu}$ which is singular at $T_{c}$ (as well as all correlation functions normalized 
as in $(\sqrt{1.2}))$. For example at one-loop order for $d=3$ the scaling function $F(z, g)$ (eq. (5.4)) is given by:

$$
\begin{aligned}
F(z, g) & =z+\frac{1}{6} z^{3}-\frac{1}{8 \pi} g z\left[\left(1+z^{2} / 2\right)^{1 / 2}-1-z^{2} / 4\right] \\
& =z+\frac{1}{6} z^{3}+\frac{1}{256 \pi} g z^{5}-\frac{1}{2^{13} \pi} g z^{7}+O\left(z^{9}\right)
\end{aligned}
$$

where the subtractions, due to the mass and coupling normalizations, are determined by the conditions (1.2). This expression is adequate for the description of the disordered phase. However when $t$ goes to zero for fixed magnetization, i.e. $m \rightarrow 0$ at $\varphi$ fixed, then $z \rightarrow \infty$ as seen in eq. (3.5). Thus all terms in the loopwise expansion become singular. In this limit one knows from eq. (3.1) that the equation of state behaves like

$$
H(M, t=0) \propto M^{\delta} \Rightarrow F(z) \propto z^{\delta} .
$$

In the framework of the $\varepsilon$-expansion the scaling relations (and thus the limiting behaviour (5.7)) are exactly satisfied order by order. Moreover the change to the variable $x \propto z^{-1 / \beta}$ (more appropriate for the regime $t \rightarrow 0$ ) gives an expression for $f(x) \propto F\left(x^{-\beta}\right) x^{\beta \delta}$ that is explicitly regular in $x=0$ (Griffith's analyticity): the singular powers of $\log x$ induced by the change of variables cancel non trivially at each order, leaving only regular corrections.

The situation changes when one deals with the perturbation theory in $d=$ 3 dimensions: scaling is not satisfied for generic values of $g$ but only at $g^{*}$. Consequently scaling properties are not satisfied order by order in $g$. In particular the change to the Widom function $f(x)$ will introduce singular powers of $x$ that violate Griffith's analyticity. An analogous problem arises if one first sums the series at $g=g^{*}$ before changing to the variable $x$. In this case the singular contributions (in the form of powers of $x$ ) do not cancel, as a result of unavoidable numerical summation errors.

Several approaches have been proposed to solve the problem of continuation to the ordered phase. A rather powerful method, motivated by the results obtained within the $\varepsilon$-expansion scheme, is based on the parametric representation [3].

Parametric representation and order dependent mapping (ODM). The problem that one faces is the following: to reach the ordered region $t<0$ one must cross the point $z=\infty$. However we know from Griffith's analyticity that $F(z) z^{-\delta}$ is regular in the variable $z^{-1 / \beta}$. This property is naturally satisfied within the parametric representation. One thus introduces an new variable $\theta$ and an auxiliary function $h(\theta)$ defined as in (4.2,4.4): the exact function $h(\theta)$ is regular near $\theta=1$ (i.e. $z=\infty$ ) and up to the coexistence curve.

The approximate $h(\theta)$ that one obtains by summing perturbation theory at fixed dimension, instead is still not regular, because the singular terms generated by the change of parametrization (4.2) at $\theta=1$ do not cancel exactly due to 
summation errors. The last step involves a Taylor expansion of the approximate expression of $h(\theta)$ around $\theta=0$ and a truncation of the expansion, to enforce regularity. A question then arises, to which order in $\theta$ should one expand? Since the coefficients of the $\theta$ expansion are in one to one correspondence with the coefficients of the small $z$ expansion of the function $F\left(z, g^{*}\right)$, the maximal power of $\theta$ in $h(\theta)$, should be equal to the maximal power of $z$ whose coefficient can be determined with reasonable accuracy. Indeed although the small $z$ expansion of $F(z)$ at each finite loop order in $g$ contains an infinite number of terms, the evaluation of the coefficients of the higher powers of $z$ is increasingly difficult. The reasons are twofold:

(i) The number of terms of the series in $g$ required to get a precise estimate of $F_{l}$ increases with $l$ because the large order behaviour sets in later.

(ii) At any finite order in $g$ the function $F(z)$ has spurious singularities in the complex $z$ plane (see e.g. eq. (5.6), $z^{2}=-2$ ) that dominate the behaviour of the coefficients $F_{l}$ for $l$ large.

In view of these difficulties one has to ensure the fastest possible convergence of the small $\theta$ expansion. For this purpose one uses the arbitrary parameter $\rho$ in eq. (4.2): one determines $\rho$ by minimizing the last term in the truncated

small $\theta$ expansion, thus increasing the importance of small powers of $\theta$ which are more precisely calculated. This is nothing but the application to this particular example of the series summation method based on ODM [49].

\section{Numerical results}

The calculation proceeds in two steps; first one determines the first coefficients of the small field expansion, then one introduces the parametric representation.

\subsection{The small field expansion}

The determination of the coefficients $F_{2 l+1}$ of the small $z$ (small field) expansion of the function $F(z)$ relies on exactly the same method as for exponents, i.e. Borel-Leroy transformation and conformal map. In figure 1 the behaviour of $F_{5}$ in terms of the Borel-Leroy parameter $b$ is displayed. Increasing flatness of the curves when $k$ increases, i.e. increasing insensitivity to the parameter $b$, supports the hypothesis that the method indeed converges.

Because the asymptotic regime sets in later when $l$ increases, the efficiency of the summation correspondingly decreases, as expected. Table 4 contains the results of [3] together with other published estimates of the coefficients of the small $z$ expansion of $F(z)$ coming from high temperature series [50,52,53, simulations [54] and derivative expansion of the Exact RG [55,56]. 
Coefficient $F_{-} 5$

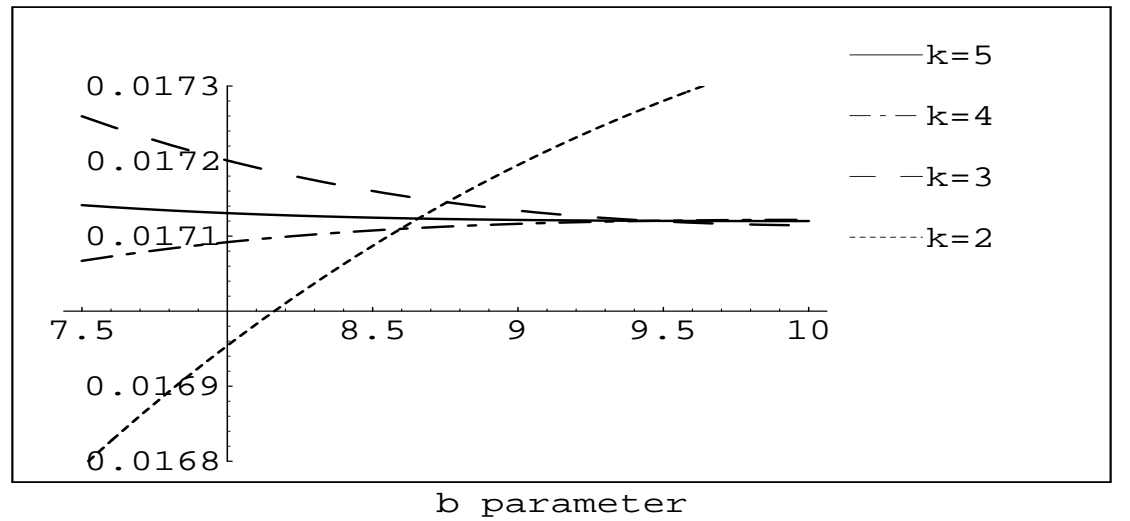

Fig. 1 The summed coefficient $F_{5}$ as a function of the Borel-Leroy parameter $b$ for successive orders $k$.

Table 1

Equation of state.

\begin{tabular}{|c|c|c|c|c|}
\hline & $g^{*}$ & $F_{5}$ & $F_{7} \times 10^{4}$ & $F_{9} \times 10^{5}$ \\
\hline$\varepsilon$-exp., [3, 2] & 23.3 & $0.0177 \pm 0.0010$ & $4.8 \pm 0.6$ & $-3.3 \pm 0.3$ \\
$\varepsilon$ - exp., [51] & $23.4 \pm 0.1$ & $0.01715 \pm 0.00009$ & $4.9 \pm 0.6$ & $-5.5 \pm 4$ \\
$d=3$, 3,2] & $23.64 \pm 0.07$ & $0.01711 \pm 0.00007$ & $4.9 \pm 0.5$ & $-7 \pm 5$ \\
HT [50] & $24.45 \pm 0.15$ & $.017974 \pm .00015$ & & \\
HT [52] & $23.72 \pm 1.49$ & $0.0205 \pm 0.0052$ & & \\
HT [53 & $23.69 \pm .10$ & $.0168 \pm 0.0012$ & $5.4 \pm 0.7$ & $-2.3 \pm 1.1$ \\
MC [54 & $23.3 \pm 0.5$ & $0.0227 \pm 0.0026$ & & \\
ERG [56] & $20.72 \pm 0.01$ & $0.01719 \pm 0.00004$ & $4.9 \pm 0.1$ & $-5.2 \pm 0.3$ \\
\hline
\end{tabular}

\subsection{Parametric representation}

One then determines by the ODM method the parameter $\rho$ and the function $h(\theta)$, as explained in section 5.2. One obtains successive approximations in the form of polynomials of increasing degree for $h(\theta)$. At leading order $h(\theta)$ is a polynomial of degree 5 , whose coefficients are given by the relations (4.7):

$$
h(\theta)=\theta\left[1+h_{3}(\rho) \theta^{2}+h_{5}(\rho) \theta^{4}\right] .
$$

For the range of admissible values for $F_{5}$ the coefficient $h_{5}$ of $\theta^{5}$ given by eq. (4.7b) 
has no real zero in $\rho$. It has a minimum instead

$$
\rho^{2}=\rho_{5}^{2}=\frac{1}{12 F_{5}}(\gamma-2 \beta) .
$$

Substituting this value of $\rho$ into expression (6.1) one obtains the first approximation for $h(\theta)$. At next order one looks for a minimum $\rho_{7}$ of $\left|h_{7}(\rho)\right|$. One finds a polynomial either of degree 5 in $\theta$, when $h_{7}$ has a real zero, or of degree 7 when it has only a minimum. It is not possible to go beyond $h_{9}(\rho)$ because already $F_{9}$ is too poorly determined.

Note that one here has a simple test of the relevance of the ODM method. Indeed, once $h(\theta)$ is determined, assuming the values of the critical exponents $\gamma$ and $\beta$, one can derive the corresponding function $F(z)$. It has an expansion to all orders in $z$. As a result one obtains a prediction for the coefficients $F_{2 l+1}$ which have not yet been taken into account to determine $h(\theta)$. The relative difference between the predicted values and the ones directly calculated gives an idea about the accuracy of the ODM method. Indeed from the values $F_{5}=0.01711, \gamma=$ $1.2398, \beta=0.3256$, one obtains

$$
F_{7}=4.83 \times 10^{-4}, \quad F_{9}=-3.2 \times 10^{-5}, \quad F_{11}=1.4 \times 10^{-7} \ldots .
$$

We see that the value for $F_{7}$ is quite close to the central value one finds by direct series summation, while the value for $F_{9}$ is within errors. This result gives us confidence in the method. It also shows that the value of $F_{9}$ obtained by direct summation contains little new information, it provides only a consistency check. Therefore the simplest representation of the equation of state, consistent with all data, is given by

$$
h(\theta)=\theta-0.76201(36) \theta^{3}+8.04(11) \times 10^{-3} \theta^{5},
$$

(errors on the last digits in parentheses) that is obtained from $\rho^{2}=2.8667$. This expression of $h(\theta)$ has a zero at $\theta_{0}=1.154$, which corresponds to the coexistence curve. The coefficient of $\theta^{7}$ in eq. (6.3) is smaller than $10^{-3}$. Note that for the largest value of $\theta^{2}$ which corresponds to $\theta_{0}^{2}$, the $\theta^{5}$ term is still a small correction. Finally the corresponding values for the $\varepsilon$-expansion are $h_{3}=-0.72, h_{5}=0.013$. These values are reasonably consistent, because a small change in $h_{3}$ can be cancelled to a large extent by a correlated change in $\rho$.

The Widom scaling function $f(x)$, eq. (3.1), can then easily be obtained numerically from $h(\theta)$ and compared with other determinations. The main disagreement with other predictions comes from the region $x \rightarrow \infty$, i.e. from the small magnetization region, where the predictions of the present method should be specially reliable. 


\subsection{Amplitude ratios}

Various amplitude ratios can then be derived from $h(\theta)$ and the values of the critical exponents determined earlier from longer series. They involve ratios of functions of $\theta$ at $\theta=0$ and at $\theta=\theta_{0}$ where $\theta_{0}$ is the zero of $h(\theta)$ closest to the origin.

Tables 5 and 6 contain a comparison of several amplitude ratios as obtained from RG, lattice calculations (High temperature series and Monte-Carlo simulations) and experiments on binary mixtures, liquid-vapour, uniaxial magnetic systems.

Table 5

Amplitude ratios.

\begin{tabular}{|c|c|c|c|c|}
\hline & $A^{+} / A^{-}$ & $C^{+} / C^{-}$ & $R_{c}$ & $R_{\chi}$ \\
\hline$\varepsilon-$ exp., [57, 46] & $0.524 \pm 0.010$ & 4.9 & & 1.67 \\
$\varepsilon-$ exp., [3, 2] & $0.527 \pm 0.037$ & $4.73 \pm 0.16$ & $0.0569 \pm 0.0035$ & $1.648 \pm 0.036$ \\
$d=3$, [47] & $0.541 \pm 0.014$ & $4.77 \pm 0.30$ & $0.0594 \pm 0.001$ & 1.7 \\
$d=3$, 3,2] & $0.537 \pm 0.019$ & $4.79 \pm 0.10$ & $0.0574 \pm 0.0020$ & $1.669 \pm 0.018$ \\
HT series [29] & $0.530 \pm 0.003$ & $4.77 \pm 0.02$ & $0.0564 \pm 0.0003$ & $1.662 \pm 0.005$ \\
MC [61] & $0.560 \pm 0.010$ & $4.75 \pm 0.03$ & & \\
bin. mix. & $0.56 \pm 0.02$ & $4.3 \pm 0.3$ & $0.050 \pm 0.015$ & $1.75 \pm 0.30$ \\
liqu. - vap. & $0.48-0.53$ & $4.8-5.2$ & $0.047 \pm 0.010$ & $1.69 \pm 0.14$ \\
magn. syst. & $0.49-0.54$ & $4.9 \pm 0.5$ & & \\
\hline
\end{tabular}

Table 6

Other amplitude ratios.

\begin{tabular}{|c|c|c|c|}
\hline & $R_{0}$ & $R_{3}$ & $C_{4}^{+} / C_{4}^{-}$ \\
\hline HT series [50, 29] & $0.1275 \pm 0.0003$ & $6.041 \pm 0.011$ & $-9.1 \pm 0.2$ \\
$d=3$, [3, 2] & $0.12584 \pm 0.00013$ & $6.08 \pm 0.06$ & $-9.1 \pm 0.6$ \\
$\varepsilon$-expansion, 3,2] & $0.127 \pm 0.002$ & $6.07 \pm 0.19$ & $-8.6 \pm 1.5$ \\
\hline
\end{tabular}

The results from the $\varepsilon$-expansion of [57,46] are obtained by direct Padé summation of each corresponding series), while the results of [3] are obtained by first summing $h(\theta)$ and then computing ratios, as explained in section 5.2. The results from the $d=3$ fixed dimension expansion of ref. 447 refer to direct summation up to $O\left(g^{5}\right)$ while the $d=3$ values of [2] again rely on the method 
explained in ref. [3]. High temperature results are taken from [50,29] (see also [58,59]). Experimental data are extracted from [60], to which we refer for more results and references. One notes the overall consistency of the results obtained by different methods.

\section{Concluding remarks}

Within the framework of renormalized quantum field theory and renormalization group, the presently available series allow, after proper summation, to determine precisely critical exponents for the $N$-vector model and the complete scaling equation of state for 3D Ising-like $(N=1)$ systems. In the latter example additional technical tools, beyond Borel summation methods, are required in which the parametric representation plays a central role. From the equation of state new estimates of some amplitude ratios have been deduced which seem reasonably consistent with all other available data.

Clearly a similar strategy could be applied to other quantities in a magnetic field, in the scaling region. Note also that an extension of the $\varepsilon$-expansion of the equation of state for $N=1$ to order $\varepsilon^{4}$ or even better $\varepsilon^{5}$, that does not seem an unrealistic goal, would significantly improve the $\varepsilon$-expansion estimates and would therefore be quite useful. Finally the present approach could be extended to systems in the universality class of the $\left(\phi^{2}\right)_{3}^{2}$ field theory for $N \neq 1$, provided expansions of the renormalized effective potential at high enough order can be generated.

More generally it must be emphasized that only a small number of universal quantities, as predicted by renormalization group, have yet been calculated. In addition to static infinite volume quantities, for which much work remains to be done, dynamic properties have not even be touched, finite size calculations would be useful for comparison with computer simulations.

Considering the increase in computer power more perturbative calculations will become feasible. What has been demonstrated here is that once the series are available, summation methods have been developed which lead to precise determinations.

Acknowledgments. The author thanks R. Guida for careful reading of the manuscript. 


\section{References}

[1] J.C. Le Guillou and J. Zinn-Justin, Phys. Rev. Lett. 39 (1977) 95; Phys. Rev. B21 (1980) 3976.

[2] R. Guida and J. Zinn-Justin, J. Phys. A31 (1998) 8103, cond-mat/9803240.

[3] R. Guida and J. Zinn-Justin, Nucl. Phys. B489 [FS] (1997) 626, hep-th/9610223.

[4] Large Order Behaviour of Perturbation Theory, Current Physics vol. 7, J.C. Le Guillou and J. Zinn-Justin eds., (North-Holland, Amsterdam 1990).

[5] An early review is K.G. Wilson and J. Kogut, Phys. Rep. 12C (1974) 75.

[6] E. Brézin, J.C. Le Guillou and J. Zinn-Justin, in Phase Transitions and Critical Phenomena vol. 6, C. Domb and M.S. Green eds. (Academic Press, London 1976).

[7] A general reference on the subject is J. Zinn-Justin, 1989, Quantum Field Theory and Critical Phenomena, in particular chap. 28 of third ed., Clarendon Press (Oxford 1989, third ed. 1996).

[8] K.G. Wilson and M.E. Fisher, Phys. Rev. Lett. 28 (1972) 240.

[9] C. Di Castro, Lett. Nuovo Cimento. 5 (1972) 69;

G. Mack, Kaiserslautern 1972, Lecture Notes in Physics vol. 17, W. Ruhl and A. Vancura eds. (Springer Verlag, Berlin 1972);

B. Schroer, Phys. Rev. B8 (1973) 4200.

[10] E. Brézin, J.C. Le Guillou and J. Zinn-Justin, Phys. Rev. D8 (1973) 2418;

J. Zinn-Justin, Cargèse Lectures 1973, unpublished, later incorporated in ref. [6];

C. Di Castro, G. Jona-Lasinio and L. Peliti, Ann. Phys. (NY) 87 (1974) 327.

[11] E. Brézin, J.C. Le Guillou and J. Zinn-Justin, Phys. Rev. D8 (1973) 434;

P.K. Mitter, Phys. Rev. D7 (1973) 2927.

[12] G. Parisi, Cargèse Lectures 1973, published in J. Stat. Phys. 23 (1980) 49.

[13] A still partially relevant reference is Phase Transitions vol. B72, M. Lévy, J.C. Le Guillou and J. Zinn-Justin eds. Proceedings of the Cargèse Summer Institute 1980 (Plenum, New York 1982).

[14] B.G. Nickel, D.I.Meiron, G.B. Baker, Univ. of Guelph Report 1977;

see also G.A. Baker, B.G. Nickel, M.S. Green and D.I. Meiron, Phys. Rev. Lett. 36 (1976) 1351;

G.A. Baker, B.G. Nickel, and D.I. Meiron, Phys. Rev. B17 (1978) 1365;

six-loop series have been published for generic values of $N$ by S.A. Antonenko and A.I. Sokolov, Phys. Rev. E51 (1995) 1894. 
[15] J.C. Le Guillou and J. Zinn-Justin, J. Physique Lett. (Paris) 46 (1985) L137;

J. Physique (Paris) 48 (1987) 19; ibidem 50 (1989) 1365.

[16] E. Brézin, J.C. Le Guillou, J. Zinn-Justin and B.G. Nickel, Phys. Lett. 44A (1973) 227;

A.A. Vladimirov, D.I. Kazakov and O.V. Tarasov, Zh. Eksp. Teor. Fiz. 77 (1979) 1035 (Sov. Phys. JETP 50 (1979) 521);

K.G. Chetyrkin, A.L. Kataev and F.V. Tkachov, Phys. Lett. B99 (1981) 147;

B101 (1981) 457(E);

K.G. Chetyrkin and F.V. Tkachov, Nucl. Phys. B192 (1981) 159;

K.G. Chetyrkin, S.G. Gorishny, S.A. Larin and F.V. Tkachov, Phys. Lett. 132B (1983) 351;

D.I. Kazakov, Phys. Lett. 133B (1983) 406;

S.G. Gorishny, S.A. Larin and F.V. Tkachov, Phys. Lett. 101A (1984) 120;

H. Kleinert, J. Neu, V. Schulte-Frohlinde, K.G. Chetyrkin and S.A. Larin, Phys. Lett. B272 (1991) 39, ibidem Erratum B319 (1993) 545.

[17] J.P. Eckmann, J. Magnen and R. Sénéor, Commun. Math. Phys. 39 (1975) 251;

J.S. Feldman and K. Osterwalder, Ann. Phys. (NY) 97 (1976) 80;

J. Magnen and R. Sénéor, Commun. Math. Phys. 56 (1977) 237;

J.-P. Eckmann and H. Epstein, Commun. Math. Phys. 68 (1979) 245.

[18] M. Ferer, M.A. Moore and M. Wortis, Phys. Rev. B8 (1973) 5205.

[19] B.G. Nickel, Physica 106A (1981) 48.

[20] J. Zinn-Justin, J. Physique (Paris) 42 (1981) 783.

[21] J. Adler, J. Phys. A16 (1983) 3585.

[22] J.H. Chen, M.E. Fisher and B.G. Nickel, Phys. Rev. Lett. 48 (1982) 630;

M.E. Fisher and J.H. Chen, J. Physique (Paris) 46 (1985) 1645.

[23] D.S. Ritchie and M.E. Fisher, Phys. Rev. B5 (1972) 2668;

S. McKenzie, C. Domb and D.L. Hunter, J. Phys. A15 (1982) 3899;

M. Ferer and A. Hamid-Aidinejad, Phys. Rev. B34 (1986) 6481.

[24] B.G. Nickel and J.J. Rehr, J. Stat. Phys. 61 (1990) 1.

[25] D. MacDonald, D.L. Hunter, K. Kelly and N. Jan, J. Phys. A25 (1992) 1429.

[26] A.J. Guttmann, J. Phys. A20 (1987) 1855;

A.J. Guttmann and I.G. Enting, J. Phys. A27 (1994) 8007.

[27] G. Bhanot, M. Creutz, U. Glässner and K. Schilling, Phys. Rev. B49 (1994) 12909.

[28] P. Butera and M. Comi, Phys. Rev. B56 (1997) 8212, hep-lat/9703018.

[29] M. Campostrini, A. Pelissetto, P. Rossi, E. Vicari, Phys. Rev. E60 (1999) 
3526, cond-mat/9905078.

[30] W. Janke, Phys.Lett. A148 (1990) 306.

[31] A.M. Ferrenberg and D.P. Landau, Phys. Rev. B44 (1991) 5081.

[32] C.F. Baillie, R. Gupta, K.A. Hawick and G.S. Pawley, Phys. Rev. B45 (1992) 10438, and references therein;

R. Gupta and P. Tamayo, Int. J. Mod. Phys. C7 (1996) 305, cond-mat/9601048.

[33] C. Holm and W. Janke, Phys. Rev. B48 (1993) 936; Phys. Lett. A173 (1993)

8, hep-lat/9605024;

K. Chen, A.M. Ferrenberg and D.P. Landau, Phys. Rev. B48 (1993) 3249;

J. Appl. Phys. 73 (1993) 5488.

[34] B. Li, N. Madras and A.D. Sokal, J. Stat. Phys. 80 (1995) 661.

[35] K. Kanaya, S. Kaya, Phys. Rev. D51 (1995) 2404.

[36] H.W.J. Blöte, E. Luijten, J.R. Heringa, J. Phys. A28 (1995) 6289, condmat/9509016;

A.L. Talapov and H.W.J. Blöte, J. Phys. A29 (1996) 5727, cond-mat/9603013.

[37] H.G. Ballesteros, L.A. Fernandez, V. Martin-Mayor and A. Munoz Sudupe, Phys. Lett. B387 (1996) 125, cond-mat/9606203. Values for $N=1$ from private communication of L.A.Fernandez.

[38] P. Belohorec and B.G. Nickel, Accurate universal and two-parameter model results from a Monte-Carlo renormalization group study, Guelph Univ. preprint $27 / 09 / 97$.

[39] S. Caracciolo, M.S. Causo and A. Pelissetto, Phys. Rev. E57 (1998) R1215, cond-mat 9703250.

[40] M. Hasenbusch, K. Pinn, S. Vinti, Phys. Rev. B59 (1999) 11471, hep-lat /9806012;

M. Hasenbusch and T. Trk, J. Phys. A 32 (1999) 6361, cond-mat/9904408.

[41] J.A. Lipa, D.R. Swanson, J. Nissen, T.C.P. Chui and U.E. Israelson, Phys. Rev. Lett. 76 (1996) 944.

[42] D.B. Murray and B.G. Nickel, unpublished Guelph report, 1991.

[43] G.M. Avdeeva and A.A. Migdal, JETP Lett. 16 (1972) 178;

E. Brézin, D.J. Wallace and K.G. Wilson, Phys. Rev. Lett. 29 (1972) 591; Phys. Rev. B7 (1973) 232.

[44] D.J. Wallace and R.P.K. Zia, J. Phys. C7 (1974) 3480.

[45] P. Schofield, Phys. Rev. Lett. 23 (1969) 109;

P. Schofield, J.D. Litster and J.T. Ho, Phys. Rev. Lett. 23 (1969) 1098;

B.D. Josephson, J. Phys. C2 (1969) 1113.

[46] J.F. Nicoll and P.C. Albright, Phys. Rev. B31 (1985) 4576. 
[47] C. Bagnuls, C. Bervillier, D.I. Meiron and B.G. Nickel, Phys. Rev. B35 (1987) 3585.

[48] F.J. Halfkann and V. Dohm, Z. Phys. B89 (1992) 79.

[49] R. Seznec and J. Zinn-Justin, J. Math. Phys. 20 (1979) 1398;

J.C. Le Guillou and J. Zinn-Justin, Ann. Phys. (NY) 147 (1983) 57;

R. Guida, K. Konishi and H. Suzuki, Ann. Phys. (NY) 241 (1995) 152;

ibidem 249 (1996) 109.

[50] S. Zinn, S.-N. Lai, and M.E. Fisher, Phys. Rev. E 54 (1996) 1176.

[51] A. Pelissetto and E. Vicari, Nucl. Phys. B519 (1998) 626, cond-mat/9711078;

ibidem B522 (1998) 605, cond-mat/9801098.

[52] T. Reisz, Phys. Lett. B360 (1995) 77.

[53] P. Butera and M. Comi, Phys. Rev. E55 (1997) 6391.

[54] M.M. Tsypin, Phys. Rev. Lett. 73 (1994) 2015.

[55] N. Tetradis and C. Wetterich, Nucl. Phys. B422 (1994) 541.

[56] T. R. Morris, Nucl. Phys. B495 (1997) 477.

[57] C. Bervillier, Phys. Rev. B34 (1986) 8141.

[58] A.J. Liu and M.E. Fisher, Physica A156 (1989) 35.

[59] A. Aharony and P.C. Hohenberg, Phys. Rev. B13 (1976) 3081; Physica 8688B (1977) 611.

[60] V. Privman, P.C. Hohenberg, A. Aharony, Universal Critical Point Amplitude Relations, in Phase Transitions and Critical Phenomena vol. 14, C. Domb and J.L. Lebowitz eds., (Academic Press 1991).

[61] M. Hasenbusch and K. Pinn, J. Phys. A31 (1998) 6157, cond-mat/9706003;

M. Caselle and M. Hasenbusch, J. Phys. A30 (1997) 4963, hep-lat/9701007;

M. Caselle and M. Hasenbusch, Nucl. Phys. Proc. Suppl. 63 (1998) 613, hep-lat/9709089.

[62] For other articles devoted to the equation of state see for example: J. Rudnick, W. Lay, D. Jasnow, Phys. Rev. E58 (1998) 2902, cond-mat/9803026;

P. Butera, M. Comi, Phys. Rev. B58 (1998) 11552, hep-lat/9805025; ibidem B60 (1999) 6749, hep-lat/9903010;

A. I. Sokolov, E.V. Orlov, V.A. Ul'kov, S.S. Kashtanov, Phys. Rev. E60 (1999) 1344, hep-th/9810082;

J. Engels, T. Scheideler, Nucl. Phys. B539 (1999) 557;

S. Seide, C. Wetterich, Nucl. Phys. B562 (1999) 524, cond-mat/9806372;

M. Strosser, S.A. Larin, V. Dohm, Nucl. Phys. B540 (1999) 654, condmat/9806103. 